\title{
Continuous Hemodiafiltration for Disseminated Intravascular Coagulation and Shock due to Amniotic Fluid Embolism: Report of a Dramatic Response
}

\author{
Yuhko Kaneko, Tohru Ogihara, Hiroto Tajima* and Fumio Mochimaru*
}

\begin{abstract}
We describe a 27-year-old woman with disseminated intravascular coagulation and shock due to amniotic fluid embolism after Caesarean section who responded well to continuous hemodiafiltration (CHDF) therapy. The effectiveness of CHDF in treating amniotic fluid embolism is also discussed.

(Internal Medicine 40: 945-947, 2001)
\end{abstract}

Key words: clinical definition, Caesarean section

\section{Introduction}

Amniotic fluid embolism (AFE) is a fatal disease. Since the precise pathophysiological mechanism of AFE has not been elucidated, no specific treatment has been established. In this article, we report a patient with AFE and propose a novel modality for the treatment of AFE.

\section{Case Report}

A previously healthy 27 -year-old para 0 , gravida 0 woman was admitted to Hiratsuka City Hospital in July 1999 for vaginal bleeding due to imminent premature labor at 28 weeks gestation. Dilatation of the cervix and cervicitis were not observed. The cause of the imminent premature labor could not be elucidated. Both mother and fetus were well conditioned by the administration of ritodrine hydrochloride. At 38 weeks of gestation, Caesarean section was performed under lumbar anesthesia with dibucaine hydrochloride because of pelvic presentation. Seventeen minutes after the start of the operation, a 2,676 $\mathrm{g}$ female infant was delivered. The neonatal Apgar score was 7 and 8 at one minute and five minutes, respectively. No placenta abnormalities were observed.

Excessive vaginal bleeding was observed despite a well- contracted uterus. Three and a half hours after the operation, the wound was resutured because of persistent non-clotting bleeding. An ultrasound examination showed no signs of bleeding in the abdominal cavity. Four hours after the operation, the patient went into shock. The hemoglobin concentration in the patient's blood had decreased from $10.7 \mathrm{~g} / \mathrm{dl}$ to $3.4 \mathrm{~g} / \mathrm{dl}$. Six and a half hours after the operation, her blood pressure was $46 /$ $22 \mathrm{mmHg}$ and she was tachycardic (140 beats per minute). Septic shock was excluded as a diagnosis because of the sudden onset of cardiovascular collapse immediately after the operation. She was treated with packed red cells, isotonic solution, and intravenous infusion of dopamine hydrochloride. Figure 1 shows her clinical course.

Laboratory investigation revealed a decrease in total protein from $6.3 \mathrm{~g} / \mathrm{dl}$ to $4.6 \mathrm{~g} / \mathrm{dl}$ and disseminated intravascular coagulation (DIC) (PT 20.0 seconds, APTT 69.0 seconds, fibrinogen $35 \mathrm{mg} / \mathrm{dl}$, FDP $>40 \mu \mathrm{g} / \mathrm{ml}$, AT-III $58.0 \%$, platelets $82,000 / \mu \mathrm{l})$. Arterial blood gas analysis revealed metabolic acidosis (base excess $-8.4 \mathrm{mEq} / \mathrm{l}$ ). The patient did not exhibit renal insufficiency or any neurological manifestations. Adult respiratory distress syndrome was not observed.

The history of sudden onset of cardiovascular collapse and DIC after Caesarean section was compatible with the clinical definition of amniotic fluid embolism proposed by Benson in 1993 (1). All other causes of DIC after a Caesarean section, such as abruption of the placenta, placenta previa, and atonic hemorrhage, were excluded. Fresh frozen plasma, platelet rich plasma, gabexate mesilate, and human anti-thrombin III were administered to treat the DIC.

About 9 hours after the Caesarean section, continuous hemodiafiltration (CHDF) using a highly permeable polyacrylonitrile hollow fiber membrane (APF-06S, Asahi Medical Co., Ltd., Japan) was performed by exchanging the extracellular fluid for artificial extracellular fluid solution at a rate of 200 $\mathrm{ml}$ per hour. The informed consent of the patient and her husband was obtained prior to the start of CHDF treatment. Fourteen hours after the CHDF was started, the patient's general condition improved, and the dose of dopamine hydrochloride

From the Department of Internal Medicine, *the Department of Obstetrics and Gynecology, Hiratsuka City Hospital, Hiratsuka

Received for publication November 1, 2000; Accepted for publication April 27, 2001

Reprint requests should be addressed to Dr. Tohru Ogihara, the Department of Internal Medicine, Hiratsuka City Hospital, 1-19-1 Minamihara, Hiratsuka, Kanagawa 254-0065 


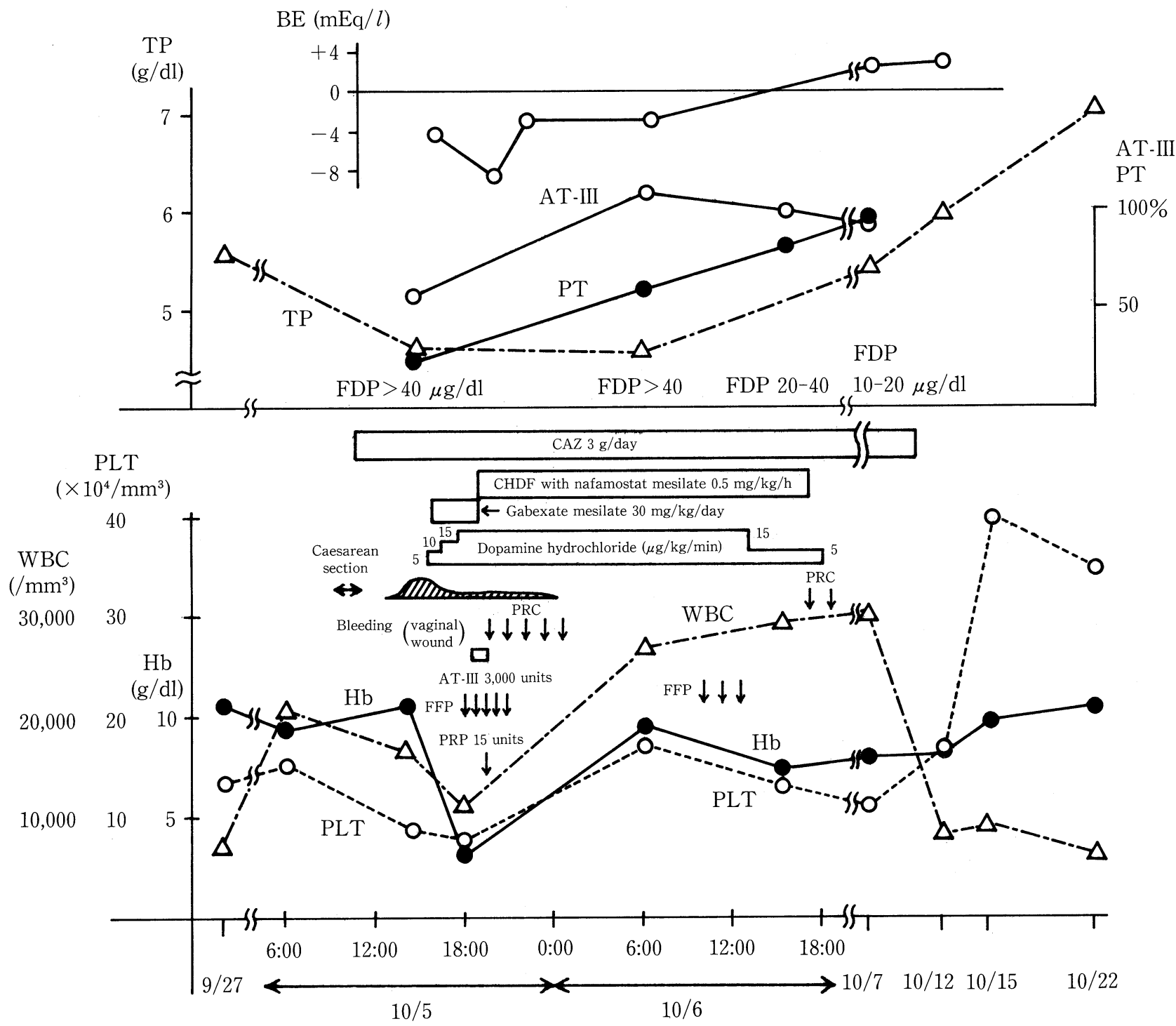

Figure 1. Clinical Course. AT-III: anti-thrombin III, BE: base excess, CAZ: ceftazidime, CHDF: continuous hemodiafiltration, FFP: fresh frozen plasma, Hb: hemoglobin, PLT: platelet, PRC: packed red cells, PRP: platelet rich plasma, PT: prothrombin time, TP: total protein, WBC: white blood cell.

was reduced from $15 \mu \mathrm{g} / \mathrm{kg} / \mathrm{min}$ to $5 \mu \mathrm{g} / \mathrm{kg} / \mathrm{min}$. Twenty-two hours after the initiation of CHDF, the procedure was discontinued because the laboratory data had improved (PT 11.0 seconds, APTT 47.7 seconds, fibrinogen $460 \mathrm{mg} / \mathrm{dl}$, FDP $20-40$ $\mu \mathrm{g} / \mathrm{ml}$, AT-III $103.0 \%$ and platelets $133,000 / \mu \mathrm{l})$. The patient subsequently improved from day to day. Twenty days after the operation, the mother and infant were discharged without any further complications.

Sialyl Tn structure (NeuAc $\alpha$ 2-6 GalNAc $\alpha$ 1-0-Ser/Thr), which is recognized by monoclonal antibody TKH-2 (2), and zinc coproporphyrin-I (3) were both measured in the maternal peripheral blood, where their source is meconium in amniotic fluid. Although their concentrations were within the normal range $(12.5 \mathrm{U} / \mathrm{ml}$ and $0.4 \mathrm{pmol} / \mathrm{ml}$, respectively), these values did not rule out AFE (4).

\section{Discussion}

AFE is a frightening and devastating disease, and the rigid diagnostic criteria for AFE is one of the reasons for its high mortality rate. Therefore, physicians must take therapeutic action as soon as AFE is suspected. With this in mind, Benson proposed a new clinical definition for AFE in 1993 (1). The new definition specifies 1) the sudden onset of cardiovascular collapse in a pregnant woman or in a patient who has delivered within the past 48 hours as defined by a pulse greater than or equal to 140 beats per minute and a diastolic blood pressure of less than or equal to $40 \mathrm{mmHg}$ or a systolic blood pressure of 
less than or equal to $60 \mathrm{mmHg}$; 2) sustained tachycardia (greater than or equal to 140 beats per minute) for 4 hours; 3) absence of other illnesses that would explain the signs and symptoms (eg, sepsis, pulmonary embolism, myocardial infarction). The definition should also distinguish between type 1 AFE with DIC (laboratory or clinical) and type 2 AFE without DIC. In this definition, DIC must include thrombocytopenia and elevated fibrin degradation products occuring within 4 hours of the initial cardiovascular collapse. Adult respiratory distress syndrome was not included in the definition.

If Benson's definition becomes widely used, the prevalence. of recognized AFE cases may increase significantly. Since the definition should permit the inclusion of a larger number of patients who survive, one would also expect a corresponding drop in the mortality rate for this condition (1). Benson's definition also permits a reduction in the AFE mortality rate because of early diagnosis and early treatment.

In the present case, the patient met the criteria for type 1 AFE and responded well to CHDF therapy. CHDF enables a continuous exchange of extracellular fluid, which contains meconium in the amniotic fluid, with an artificial extracellular solution and can remove many substances with a molecular weight (MW) of up to $30 \mathrm{kD}$ to $40 \mathrm{kD}(5)$. Removal of cytokines, such as interleukin-6 (MW $21 \mathrm{kD}$ ) and interleukin-8 (MW $8 \mathrm{kD}$ ), using CHDF is a reasonable method for treating hypercytokinemia, which can cause multiple organ failure (MOF) (5).

Consideration must be given to which is better for the treatment of AFE, CHDF or continuous hemofiltration (CHF). CHF is more effective at eliminating high molecular weight substances than CHDF; however, CHDF is more effective than $\mathrm{CHF}$ for the treatment of metabolic acidosis. Therefore, we selected CHDF for the treatment of AFE.

Treatment for DIC consists of (1) treatment for the causative disease and (2) general treatment for the symptoms of DIC. Treatment for shock consists of (3) treatment for the causative disease and (4) general treatment for the symptoms of shock. The use of CHDF to treat AFE followed by DIC and shock enables all of the above treatments to be provided (1)+ (2)+(3) +(4) because CHDF can eliminate amniotic fluid from the maternal blood stream and can correct the hyper-cytokinemia and metabolic acidosis produced by amniotic fluid leakage.

CHDF must be continued for 1 to 3 weeks in patients with MOF due to pancreatitis, because the damaged pancreas produces many humoral factors for a long period. However, only short-term CHDF may be needed in AFE, because the total amount and duration of amniotic fluid leakage into the maternal blood stream are limited.

Since AFE is a rare condition, no single institution has sufficient experience to assess the effectiveness of CHDF therapy. However, we think that CHDF may be a novel modality for the treatment of AFE.

Acknowledgements: We wish to thank Dr. Hidekazu Oi, Department of Obstetrics and Gynecology, Hamamatsu University School of Medicine, Hamamatsu, Shizuoka, Japan, for measuring the zinc coproporphyrin-1 and NeuAc $\alpha$ 2-6 GalNAc $\alpha$ 1-0-Ser/Thr (sialyl Tn).

\section{References}

1) Benson MD. Nonfatal amniotic fluid embolism-Three possible cases and a new clinical definition. Arch Fam Med 2: 989-994, 1993.

2) Kobayashi H, Ohi H, Terao T. A simple, noninvasive, sensitive method for diagnosis of amniotic fluid embolism by monoclonal antibody TKH2 that recognizes NeuAc $\alpha$ 2-6 GalNAc. Am J Obstet Gynecol 168: 848$853,1993$.

3) Kanayama N, Yamazaki T, Naruse H, Sumimoto K, Horiuchi K, Terao T. Determining zinc coproporphyrin in maternal plasma-A new method for diagnosing amniotic fluid embolism. Clin Chem 38: 526-529, 1992.

4) Kobayashi $\mathrm{H}$, Ooi $\mathrm{H}$, Hayakawa $\mathrm{H}$, et al. Histological diagnosis of amniotic fluid embolism by monoclonal antibody TKH-2 that recognizes NeuAc $\alpha$ 2-6 GalNAc epitope. Hum Pathol 28: 428-433, 1997.

5) Hirasawa H, Sugai T, Ohtake Y, Oda S, Matsuda K, Kitamura N. Blood purification for prevention and treatment of multiple organ failure. World J Surg 20: 482-486, 1996. 